\title{
La Atención Primaria de Salud: Un Programa Viable para las Comunidades Indígenas
}

* Licenciado en Enfermerla. Fundación para las Comunida. des Colombianas - Funcol.
Francisco A. Zambrano $S .^{*}$ César Méndez Lizarazo*

"No son las metas y objetivos de un programa comunitario lo que lo hace vital o viable sino más bien la visión no escrita y cambiante, compartida por

los miembros del Programa y la Comunidad a medida que cambian $y$ evolucionan juntos."

David Werner

\section{INTRODUCCIÓN}

La Fundación para las Comunidades Colombianas - Funcol-, ha venido desarrollando desde hace varios años su programa de Atención Primaria de Salud en Comunidades Indígenas de la Orinoquia y Zona Andina Colombianas, desde una perspectiva multidisciplinaria, ampliando permanentemente su marco de referencia teórico-práctico.

Las modificaciones introducidas al programa, provienen entre otras cosas, de los aportes y situación particular presentadas por las comunidades beneficiadas, por las variantes que presentan las políticas del Estado en el campo de la salud y las que Funcol considera aplicables a cada uno de las etnias, en sus respectivas zonas geográficas.

A través de este proceso, hemos logrado determinar los aspectos de la problemática de salud de cinco grupos étnicos distintos e integrar conceptos nuevos a las visiones que se tienen en este campo en la esfera nacional e internacional.

Igualmente hemos podido obtener una visión de conjunto de la dinámica de estos grupos y por consiguiente determinar los logros y limitaciones que conlleva un programa de esta naturaleza.

También nos hemos enriquecido con aportes de personas y entidades que desarrollan programas similares, bien sea en comunidades indígenas o en otros sectores de población, a través de la realización y participación en foros, talleres y seminarios tanto a nivel local, regional, nacional e intemacional. 
El mejoramiento de las condiciones de salud de las comunidades vinculadas al programa, ha dependido de múltiples factores, entre los cuales ha contado la voluntad de sus integrantes de resistir culturalmente, reforzando en primer término su cohesión interna $\mathrm{y}$ ligándose a procesos organizativos, cuya dinámica se ha visto en los últimos años en el plano nacional.

Con base en estas consideraciones, el programa se ha convertido en recurso de primera mano, para suplir la prestación de servicios a nivel comunitario, exigiendo del estado la ampliación de la cobertura y una mejor atención a sus necesidades. Desde la iniciación de nuestro programa fue nuestro propósito dejar en manos de la comunidad, una alternativa abierta, que incluía en primer término el reforzamiento de su confianza para resistir la colonización y exigir del Estado el cumplimiento de sus obligaciones en materia de tierras y salud.

Nuestro programa entendió que era necesario tener en cuenta los recursos existentes en cada comunidad y con ellos y otros provenientes de la medicina institucional, solucionar las necesidades más urgentes de salud.

El reto se concentró a mostrar eficacia y de esta manera contribuir a elevar el nivel de corciencia en cuanto a autonomia y derechos de los indígenas. En estas condiciones, cada comunidad entró en un nuevo nivel que le permitió abandonar su condición de sujetos pasivos de acciones esporádicas provenientes de las agencias del Estado, entrando a participar activamente en la planeación y ejecución de los programas requeridos, partiendo de sus propios recursos y de aquellos propios de la cultura occidental aplicables a su medio.

\section{CONSIDERACIONES GENERALES}

Teniendo en cuenta que toda comunidad o grupo humano posee recursos de salud que tradicionalmente ha utilizado para sobrevivir y habiéndonos enfrentado a dificultades de índole cultural al tratar de implementar programas sanitarios, nos vimos en la necesidad de profundizar en su conocimiento e inventariar y sistematizar los recursos, tratando simultáneamente de reforzar y ampliar su utilización, para impedir que desaparezcan con la expansión de las acciones propias de la medicina institucional.

Uno de los principales problemas que enfrentan las comunidades indígenas del país y particularmente las vinculadas a nuestro programa, es la reducción de sus territorios, ya que la mayoría de ellas estan ubicadas en zonas "disponibles" para la colonización, propiciando el contacto permanente con poblaciones

Cadernos de Saúde Pública, RJ, 7(1): 104-114, jan/mar, 1991. 
campesinas desplazadas, también víctimas de procesos que han implicado la pérdida de su habitat. Por consiguiente han sufrido situaciones de desarraigo, malnutrición y alta morbimortalidad.

Esto les ha traído implicaciones negativas sobre la disponibilidad de recursos de caza, pesca, recolección y calidad de sus áreas de cultivo, trayendo como consecuencia una incidencia directa sobre las condiciones de salud y nutrición, que se manifiestan actualmente en la elevación considerable de las tasas de morbimortalidad generada por las enfermedades propias de la pobreza y el abandono, ubicadas por encima de las establecidas a nivel nacional.

El saber médico indígena ha evolucionado a través de los años y la presencia de sus especialistas al igual que la vigencia de sus prácticas depende de la intensidad de los procesos descritos. La solidéz de su organización interna y la fuerza de su cultura son aspectos que juegan papel importante en la trasmisión y difusión de estos conocimientos.

Sin embargo, en algunas etnias éste saber se viene diluyendo y faltan los especialistas, dando lugar a un mayor desorden intemo pues ellos además de cumplir las funciones de vigilantes de la salud del grupo, regulan las relaciones sociales y ambientales y los reviste de poder y prestigio tanto interna como externamente.

El ejercicio de los Shamanes, Karecas, Médicos o Brujos, se ha visto limitado y en algunos casos perseguidos. Esto ha reducido aún más el número de sus seguidores y desestimulado a los futuros médicos tradicionales. La introducción de nuevos modelos de organización interna ha venido suplantándolos y son contados los casos en que han podido articularlo, ya que se manteniene el enfrentamiento por el control del grupo.

Con relación al proceso de formación de estos personajes, señalamos que depende de su especialización u oficio, existiendo entre los maestros y los aprendices relaciones de parentesco y en contados casos por actitudes o por señalamiento o indicación natural.

La duración del periodo de aprendizaje es indefinida, dependiendo de los progresos que demuestre en la práctica diaria. En todos los casos incluye ayunos, abstinencia sexual, prolongadas vigilias, comer sin sal, desplazamientos permanentes a los sitios de interés donde se reconocen los recursos de flora, fauna y minerales propios de su oficio y donde aprenden además de su uso, la manera de recolectarlos y tratarlos para su trabajo futuro.

Con relación a la enfermedad, su conocimiento se deriva de la relación signo-síntoma-ambiente, sin 
adentrarse en el conocimiento interno del cuerpo, aunque no sobra decir, existen conocimientos amplios sobre éste tema incertos en cada una de las culturas.

Tenemos que recalcar que a pesar de las dificultades por las que atraviesa la práctica tradicional de salud, este proceso de conocimiento empírico no sistematizado, que se ha quedado sin mayor profundización y que comienza a ser superado por otros modelos culturales, sigue aportando elementos de análisis y mantiene su vigencia en amplios sectores no solo de la población indígena sino en otros culturalmente diferentes.

En resumen, el análisis de esta estructura del conocimiento y su dinámica social nos conduce necesariamente a cambiar los conceptos de combatirlos o suprimirlos, ya que poseen aspectos positivos de salud que los hacen efectivos al integrarlos a los programas de salud trazados y se permite de esta forma que cada comunidad los identifique y apoye decididamente.

A nivel estatal, con el establecimiento de niveles jerarquizados en la atención de salud, la utilización de prácticas tradicionales que proceden de diferentes culturas, nunca fueron consideradas. El saber médico occidental rechazó, con una postura colonial, la existencia de prácticas de salud, que fueron cosideradas irracionales y reprimibles.

La presencia de programas estatales en los diferentes grupos indígenas, está mediatizada por la intensidad del fenómeno propio de la colonización y los conflictos socio-políticos que éste genera. Esto ha afectado tanto la oferta como la demanda de servicios en mayor o menor grado en las diferentes zonas geográficas.

La cobertura de los programas gubernamentales se ha ampliado a otros grupos de población, especialmente al sector campesino que tiene una mayor capacidad de demanda, en tanto que a las comunidades indígenas se les ha mantenido relegadas, pese a los esfuerzos por incrententar los servicios de salud a estos sectores de población.

Este fenómeno se genera por su forma de penetración, metodología, objetivos, premisas, planificación, los niveles culturales de comunicación; componentes que matizan el distanciamiento y caracterizan la relación de tipo vertical entre los programas institucionales $\mathrm{y}$ las espectativas de las distintas comunidades.

La persistencia de esta visión etnocéntrica, que genera la actitud paternalista de las acciones y que refuerza el desconocimiento por parte de los agentes externos de la cosmovisión indígena, frente al problemas de la salud y la enfermedad, los coloca en desventaja frente a la elección de las posibles soluciones.

A menudo los programas estatales no se ajustan a la realidad que vive la comunidad, pues obedecen 
a planes trazados, con una visión urbana, para toda la población. Además, debido a la estructura de organización política del Estado estos se ven afectados frecuentemente para su implementación por trabas y discontinuidades burocráticas.

Por otra parte, la infraestructura sanitaria sigue siendo insuficiente y en la mayoría de estas comunidades solo se conocen acciones esporádicas con resultados poco duraderos, debido a que las condiciones ambientales favorecen la persistencia de enfermedades, las cuales serían facilmente controlables a través de acciones preventivas, proyectadas en el tiempo, modificando de esta forma su comportamiento epidemiológico.

En general, las comunidades se han convertido en simples receptoras de servicios, de manera que no colaboran en forma sistemática en el diagnóstico, planeación, ejecución y evaluación de los programas, generando en algunos casos desinterés y en otros franco rechazo a las acciones propuestas.

Solo en los últimos años y muy tímidamente han variado algunos de estos criterios, gracias a la movilización de las comunidades, a los estudios antropológicos y a la investigación de entidades de caracter nacional e internacional como la OMS.

Por nuestra parte el programa, basado en el respeto por la cultura indígena y la convivencia participante ha venido canalizando sus concepciones, actitudes, valores y prácticas médicas tradicionales, tratando de superar el criterio de imposición de elementos tecnológicos universales, con el ánimo de encontrar nuevos caminos que nos permitan abordar la problemática de salud y enfermedad al incorporarlos simultáneamente.

En resumen el Programa de Funcol ha venido delineando el perfil de las condiciones de salud, la persistencia de prácticas médicas tradicionales, su relación con la medicina institucional, otras prácticas culturales y recursos, concretando criterios sobre la forma como se deben prestar los servicios institucionales, para generar el cambio en la actitud asumida por la comunidad, mejorar los programas y modificar conjuntamente las condiciones de salud de cada comunidad participante.

\section{PROPÓSITOS Y OBJETIVOS}

1. Contribuir al mejoramiento del nivel de salud de los grupos étnicos: U'wa (Tunebo), Sicuani (Guahibo), Dejá (Piapóco), Wanome (Cuiba), Sáliba y Nasa (Paez) de la Orinoquia y zona Andina colombiana, vinculados al programa, mediante la profundización en el conocimiento de su problemática y 
prácticas culturales e impulsando acciones para que la comunidad se las vaya apropiando en la medida de su desarrollo.

2. Rescatar y velar por la conservación de las prácticas tradicionales de cada grupo, evitando en lo posible la sustitución por las provenientes de la medicina occidental, facilitando el intercambio donde fuese posible.

3. Estimular la participación comunitaria en todas las etapas contempladas para el desarrollo del progra$\mathrm{ma}$, com miras a que sea integrado al proceso de autogestión.

4. Poner al servicio de la comunidad, elementos propios de la medicina occidental que den respuesta a sus necesidades y espectativas, a través de agentes pertenecientes a cada comunidad con acciones que se emarquen dentro de los lineamientos de mutuo respeto y apoyo.

\section{CRITERIOS ORGANIZATIVOS}

A partir del diagnóstico establecido a través de los estudios de factibilidad antropológica y de la determinación de los perfiles epidemiológicos con participación comunitaria, llegamos al lineamiento de los criterios básicos para la implementación amplia del programa de salud.

1. Dado que las barreras culturales existentes, se convertían en serio obstáculo para el logro de los objetivos propuestos, se hacía necesario contar con canales de aproximación que solo podían ser aportados por la misma comunidad y para ello se procedió internamente a la selección de personas que cumplieran esta función, procediendo a su capacitación, mediante el adietramiento elemental para niveles de escolaridad muy bajos, pero que a la ves fueran suficientes para influir eficazmente en la modificación de la morbimortalidad presente en cada asentamiento.

2. El conjunto de acciones impulsadas hasta el momento no se orientan exclusivamente a los aspectos de salud. Contemplan la promoción y organización interna, representación y gestión ante las entidades gubernamentales, religiosas y privadas, reforzamiento de programas de producción y educación además de las actividades específicas de atención a la enfermedad en los niveles de prevención, reparación y rehabilitación.

3. El manejo operativo del programa se ha venido entregando paulatinamente a la comunidad, de acuerdo a las condiciones particulares de cada asentamiento, manteniendo durante el tiempo que sea necesaria la asistencia y evaluación. 
Los criterios expuestos nos muestran que, dentro de cada étnia, existen comunidades con diversos grados de desarrollo, que afecta en distinta medida el progreso del programa.

Lo más relievante, es que aquellas comunidades que han sido duramente golpeadas por el proceso de colonización, con las consecuencias obvias de pérdida de su identidad cultural, pérdida de su lengua, costumbres, formas organizativas y que no han podido establizarse, han tenido mayor dificultad para participar en el programa, que aquellas que aún conservan aspectos importantes de la cultura y especialmente el recurso tradicional de salud, o aquellas que se han asimilado como comunidades campesinas, en donde los resultados son más halagadores.

El modelo de administración y manejo del programa implementado en cada asentamiento ha fomentado la autoayuda y en general estimula el aporte de todos para su sostenimiento. Se han presentado algunas dificultades en algunas zonas y en comunidades en particular, donde agentes extemos han impulsado acciones caritativas y donaciones que han generado en sus beneficiarios actitudes indigentes.

Dado que las relaciones entre la comunidad, la entidad responsable y sus ejecutores se han basado en el respeto por la cultura y sus tradiciones, se ha facilitado, en gran medida, el rescate, inventario, conservación y recopilación de una buena parte de las prácticas tradicionales de salud, haciendo la complementación con los aportes propios de la medicina de occidente.

No despreciamos los avances tecnológicos; por el contrario, en la medida del desarrollo y aceptación por parte de la comunidad, se han puesto a su servicio, como en el caso concreto de los estudios epidemiológicos donde se han practicado pruebas que sólo son factibles de ejecutar en el medio hospitalario y que sólo de esta manera han podido llegar a sus propios asentamientos, facilitando y estimulando el uso racional de cada uno de los recursos puestos al servicio de la comunidad.

\section{DEFINICIÓN DE SUB-PROGRAMAS}

A partir de las recomendaciones formuladas por las OMS y la reglamentación existente en el País, para la organización de Servicios de Salud en Atención Primaria, hemos definido las áreas o sub-programas con fines exclusivos de planeación y ejecución, pues el programa como tal exige interrelación estrecha en cada uno de ellos y no es posible concebirlos funcionando aisladamente. 


\section{Sub-Programa de Adiestramiento}

Es la capacitación permanente de miembros de la comunidad en técnicas de Atención Primaria en salud, técnicas paramédicas sencillas, de tal manera que logren atender los problemas de salud que se presentan con mayor frecuencia. Esta capacitación se adelanta en cada una de las zonas geográficas y en la comunidad o sitio que hayan escogido para tal fín.

El participante debe identificar las enfermedades de alta prevalencia en su comunidad, tanto desde el punto de vista cultural como institucional y aprende a aplicar medidas preventivas, de protección y restauración de la salud, a partir de los recursos propios y de los aportados por el programa.

La capacitación contempla temas sobre anatomía, fisiología, primeros auxilios, inyectología, enfermedades frecuentes, tratamientos y cuidados, manejo de estadísticas vitales, uso y cuidados de los medicamentos, nutrición, higiene, saneamiento ambiental y zoonosis.

El aprendizaje se implementa a partir de las situaciones presentes en el sitio de capacitación, se identifican necesidades y se plantean posibles soluciones. Este proceso se refuerza con los hallazgos de los estudios efectuados y las observaciones directas, empleando en la solución todos los recursos del medio, ayudas didácticas elaboradas para el programa y los recursos externos estrictamenten necesarios.

La evaluación del capacitado es permanente, la comunidad vigila sus actividades y valora los resultados. En cada visita del equipo ejecutor se evalua la participación comunitaria así como el trabajo desarrollado por el trabajador de salud, se revisan conocimientos y prácticas, se establecen correctivos y se sistematiza el trabajo efectuado en cada período.

\section{Sub-Programa de Atención en Salud}

Se ha definido como el conjunto de acciones de promoción, prevención, reparación y rahabilitación de la salud, ejecutadas en la comunidad por los trabajadores de salud, médicos tradicionales y agentes externos incluídos los ejecutores del programa.

Contempla los siguientes componentes:

\section{Atención Infantil}

Orientada a la prestación de primeros uxilios, identificación da EDA, Poliparasitismo intestinal, enfermedades respiratorias, infecciones de piel, desnutrición y deshidratación, cuidados y remisiones a los centros de atención de mayor complejidad.

Cadernos de Saúde Páblica, RJ, 7(1): 104-114, jan/mar, 1991. 
Aparte de las actividades correspondientes al grupo infantil: identificación de paludismo, tuberculosis, hipertensión, cuidados, administración delegada de tratamientos y remisiones.

\section{Atención Materna}

A pesar de la importancia que tiene este componente, ha requerido de un manejo cuidadoso en lo referente a embarazo, parto y cuidado del recien nacido, por los celos culturales que dificultan su penetración. Sin embargo, actividades relacionadas con el control del embarazo, señales de peligro, molestias, problemas nutricionales y anémia, se han podido desarrollar sin contratiempo. Progresivamente ha mejorado la aceptación y ya se ha pedido en algunas comunidades y zonas la inclusión del tema en la capacitación de los trabajadores de salud.

\section{Vacunación}

El programa amplio de inmunizaciones impulzado por el Ministerio de Salud a través de los Servicios Seccionales se ha apoyado constantemente y se ha buscado su reforzamiento y ampliación de la cobertura en tanto que a nivel comunitario se ha impartido ilustración permanentemente, obteniéndose buena acogida para el programa en la mayoría de los asentamientos.

\section{Vigilancia Epidemiológica e Información Estadística}

Mediante la aplicación sistemática de instrumentos sencillos de morbimortalidad y natalidad, el trabajador de salud mantiene actulizada la información de su respectiva comunidad. Además da aviso oportuno en caso de epidemias o casos raros a los centros de atención más cercanos.

\section{Sub-Programa de Boticas Rotatorias o Microdis- pensarios}

Constituye un elemento de apoyo a las diferentes actividades de salud que impulsa el programa. Es manejado por el trabajador de salud y controlado por la comunidad.

Se busca con él, que cada comunidad llegue al autosostenimiento a partir de una entrega inicial, compuesta por un equipo básico y los medicamentos de uso más frecuente que ha aprendido a manejar el trabajador de salud en el transcurso de la capacitación.

La implementación de este subprograma, requiere de un análisis detenido, ya que en la mayoría de las 
comunidades se emplea una cantidad enorme de medicamentos que han sido intruducidos a lo largo del contacto con otros grupos culturales, haciendo de la automedicación un problema cotidiano y serio para el manejo de las patologías propias de cada zona.

Debe incluir la educación a la comunidad, de tal manera que en corto tiempo se llegue al uso racional de estos productos y se conforme un listado de medicamentos que ofrezca entre otras características las siguientes: 1. Fácil conservación. Debe resistir al deterioro propio de ambientes cálidos $\mathrm{y}$ fríos húmedos; 2. Fácil adquisición y bajo precio; 3 . Reducir y estandarizar el número de medicamentos que se utilizan en cada comunidad; 4 . Lograr la cobertura con medicamentos de eficacia y seguridad comprobadas; 5 . Adelantar los suministros y la adquisición de medicamentos con nombres genéricos o comerciales estables para evitar confusiones en relación a la concentración, la dósis, indicaciones y contraindicaciones.

Por las condiciones socio-culturales y la falta de experiencia en el manejo y control de este tipo de recursos (porque generalmente cada familia o persona maneja libremente y de acuerdo a sus convicciones o experiencias este mercado), hemos tenido dificultades, que progresivamente se van superando en la medida que se fortalece el programa en su conjunto y se asesora permanentemente a la comunidad corrigiendo los errores en el menejo y control.

Cada comunidad ha venido estableciendo mecanismos para la implementación de este subprograma, yendo desde los aportes familiares anuales, cuotas voluntarias, aportes de cosechas, pagos de los servicios, búsqueda de ayudas externas, aportes en medicamentos o pago en especie de los mismos y canalización de recursos económicos a través de actividades culturales.

\section{Sub-Programa de Saneamiento Ambiental}

Comprende el conjunto de acciones teóricas y prácticas encaminadas a solucionar los problemas relacionados con el medio ambiente y que afectan directamente el estado de salud de la comunidad.

Considera entre otros los siguientes componentes: Agua, eliminación de excretas y basuras, mejoramiento de las viviendas, higiene y zoonosis.

\section{REFERÊNCIAS BIBLIOGRÁFICAS}

ARBElÅEZ AlBORNOZ, Camilo. Programa de Atención Primaria de Salud para Comunidades Indígenas. Funcol, Bogotá, 1979.

Cadernos de Salde Pública, RJ, 7(1): 104-114, jan/mar, 1991. 
ALZATE, Maria Cristina. Informe de Trabajo de Campo - Funcol. Bogotå, 1979.

BASTIDAS, Roger. Antropologla Aplicada. Amorrortu Editores. Buenos A ires, Argentina, 1971.

CANGUILHEM, Georges. Lo normal y lo Patolbgico. Siglo XXI Editores. 7: Édición. México, 1986.

CHA VEZ, Adolfo y MARTINEZ, C. Nutrición y desarrollo Infantil. Nueva Editorial Intereamericana. México, 1979.

ESCALANTE ANGULO, Carlos. Investigación Socio-médica. Sexta Edición ampliada y corregida. Ediciones Tercer Mundo. Bogotá, 1985.

FERGUSSON, Guilermo. Esquema crftico de la Medicina en Colombia. Bogotá, 1979.

GEOTCHEUS, Janelle. Los restrictivos sistemas de atención de salud en las sociedades opulentas. Contact: 52. Ginebra-Suiza, 1982.

KINGMA, Stuart. Medicamentos esenciales al alcance de la población. Contact: 48. Ginebra Suiza, 1981.

KNOK, E. G. La epidemiologla en la planificación de la atención a salud. Siglo XXI Editores. Mexico, 1981.

McKEOWN, Thomas. El papel de la medicina: Sueño, espejismo o némesis?. Siglo XXI Editores. Primera Edición en Español. Mexico, 1982.

MENDES GONCALVES, Ricardo Bruno. Medicina e Historla: Raices sociales del trabajo médico. Siglo XXI Editores. Primera edición en Español. Mexico, 1984.

MINISTERIO DE SALUD. Dirección de Participación de la Comunidad. Marco Programático para la prestación de Servicios de Salud a Comunidades Indfgenas. Bogotá, 1978.

MINISTERIO DE SALUD. Dirección de Investigaciones. Primera Reunión sobre Medicina Tradicional, Bogotá, 1981.

ORGANIZACION MUNDIAL DE LA SALUD. Unicef. AlmaAta. Atencion Primaria de Salud. Publicación Cientifica OMS. Ginebra-Suiza, 1978.

PEREZ TAMAYO, Ruy. Enfermedades viejas y enfermedades nuevas. Siglo XXI Editores. Primera Edición. Mexico, 1985.

VASCO URIBE, Alberto. Salud, Medicina y Clases Sociales. Editorial La Pulga. Medellfin, 1975.

VASCO URIBE, Alberto. Enfermedad y Sociedad. Ediciones Hombre Nuevo. Medellin, 1979.

WERNER, David. Asistencia Sanitaria y Dignidad Humana. Contact: 42. Ginebra. Suiza, 1980. 